\title{
The Earth's Core: An Approach from First Principles
}

\author{
G. David Price ${ }^{1}$, D. Alfè ${ }^{1,2}$, L. Vočadlo ${ }^{1}$, and M.J. Gillan ${ }^{2}$ \\ ${ }^{1}$ Research School of Earth Sciences, Birkbeck and University College London, Gower Street, \\ London WC1E 6BT, UK. \\ ${ }^{2}$ Department of Physics and Astronomy, University College London, Gower Street, London \\ WC1E 6BT, UK.
}

\begin{abstract}
The Earth's core is largely composed of iron (Fe), alloyed with less dense elements such as sulphur, silicon and/or oxygen. The phase relations and physical properties of both solid and liquid Fe-alloys are therefore of great geophysical importance. As a result, over the past fifty years the properties of $\mathrm{Fe}$ and its alloys have been extensively studied experimentally. However, achieving the extreme pressures (up to $360 \mathrm{GPa}$ ) and temperatures $(\sim 6000 \mathrm{~K})$ found in the core provide a major experimental challenge, and it is not surprising that there are still considerable discrepancies in the results obtained by using different experimental techniques. In the past fifteen years quantum mechanical techniques have been applied to predict the properties of Fe. Here we review the progress that has been made in the use of first principles methods to study Fe and its alloys, and as a result of these studies we conclude: (i) that pure Fe adopts an hexagonal close packed structure under core conditions and melts at $\sim 6200 \mathrm{~K}$ at $360 \mathrm{GPa}$, (ii) that thermodynamic equilibrium and observed seismic data are satisfied by a liquid $\mathrm{Fe}$ alloy outer core with a composition of $\sim 10$ mole $\% \mathrm{~S}$ (or $\mathrm{Si}$ ) and $8 \mathrm{~mole} \% \mathrm{O}$ crystallising at $\sim 5500 \mathrm{~K}$ to give an Fe alloy inner core with $\sim 8$ mole $\% \mathrm{~S}$ (or Si) and 0.2 mole $\% \mathrm{O}$, and (iii) that with such concentrations of $\mathrm{S}$ (or $\mathrm{Si}$ ), an Fe alloy might adopt a body centred cubic structure in all or part of the inner core. In the future the roles of $\mathrm{Ni}, \mathrm{C}, \mathrm{H}$ and $\mathrm{K}$ in the core need to be studied, and techniques to predict the transport and rheological properties of Fe alloys need to be developed.
\end{abstract}

\section{Introduction}

Knowing about the nature of the Earth's core lies at the heart of understanding the state of our planet. Today, we believe that the core is the source of the Earth's magnetic field, and that heat-flow from the core contributes significantly to driving mantle convection, and hence ultimately contributes to plate tectonics and the resulting evolution of the planet's surface. What we know of the details of the core's structure comes largely from seismology. Although previously inferred to exist from a knowledge of the mass and moment of inertia of the Earth, the presence of the Earth's core was only firmly established from seismology by Oldham in 1906. Further seismological study enabled Gutenberg (1913) to determine the depth of the core-mantle boundary (CMB) to be $\sim 2890 \mathrm{~km}$, and in 1936 Lehmann discovered that there existed an inner core (with a radius now known to be $\sim 1220 \mathrm{~km}$ ). It was not until 35 years later that Dziewonski and Gilbert (1971) definitely proved that the inner core was a solid region within the surrounding liquid outer core. Seismology has subsequently shown that the inner core is anisotropic, with seismic waves travelling faster parallel to the poles than in the equatorial plane (e.g. Creager, 1992), and most recently, seismic measurements have been interpreted as showing that the Earth's solid inner core is even more structurally complex. The detailed interpretations of the data differ, but all workers (e.g. Song and Helmberger, 
1998; Ishii and Dziewonski, 2002; Beghein and Trampert, 2003) conclude that the inner core exhibits a significant degree of layering, which may either reflect the changing history of core crystallisation and deformation, or the occurrence of an unidentified change in the coreforming phases.

The fact that the core is largely composed of Fe was firmly established as a result of Birch's (1952) analysis of mass-density/sound-wave velocity systematics. Today we believe that the outer core is about 6 to $10 \%$ less dense than pure liquid Fe, while the solid inner core is a few percent less dense than crystalline Fe (e.g. Poirier, 1994a). From cosmochemical and other considerations, it has been suggested (e.g. Poirier, 1994b; Allègre et al., 1995; McDonough and Sun, 1995) that the alloying elements in the core might include $\mathrm{S}, \mathrm{O}, \mathrm{Si}, \mathrm{H}$ and $\mathrm{C}$. It is also probable that the core contains minor amounts of other elements, such as $\mathrm{Ni}$ and $\mathrm{K}$. The exact temperature profile of the core is still controversial (e.g. Alfè et al., 2002a), but it is generally held that the inner core is crystallising from the outer core as the Earth slowly cools, and that (as a result of the work outlined below) core temperatures are in the range $\sim 4000-5500 \mathrm{~K}$, while the pressure at the centre of the Earth is $\sim 360 \mathrm{GPa}$.

Before a full understanding of the chemically complex core can be reached, it is necessary to understand the properties and behaviour at high pressure $(P)$ and temperatures $(T)$ of its primary constituents, namely metallic Fe. Experimental techniques have evolved rapidly in the past years, and today using diamond anvil cells or shock experiments the study of minerals at pressures up to $\sim 200 \mathrm{GPa}$ and temperatures of a few thousand Kelvin is possible. These studies, however, are still far from routine, and results from different groups are often in conflict (see for example reviews by Poirier, 1994b; Shen and Heinz, 1998; Stixrude and Brown, 1998; Boehler, 2000). As a result, therefore, in order to complement these existing experimental studies and to extend the range of pressure and temperature over which we can model the Earth, computational mineral physics has, in the past decade, become an established and growing discipline.

Within computational mineral physics a variety of atomistic simulation methods (developed originally in the fields of solid state physics and theoretical chemistry) are used. These techniques can be divided approximately into those that use some form of interatomic potential model to describe the energy of the interaction of atoms in a mineral as a function of atomic separation and geometry, and those that involve the approximate solution of Schrödinger's equation to calculate the energy of the mineral species by quantum mechanical techniques. For the Earth sciences, the accurate description of the behaviour of minerals as a function of temperature is particularly important, and computational mineral physics usually uses either lattice dynamics or molecular dynamics methods to achieve this important step. The relatively recent application of all of these advanced condensed matter physics methods to geophysics has only been possible by the very rapid advances in the power and speed of computer processing. Techniques, which in the past were limited to the study of structurally simple compounds, with small unit cells, can today be applied to describe the behaviour of complex, low symmetry structures (which epitomise most minerals) and liquids.

In this paper, we will focus on recent studies of Fe and its alloys, which have been aimed at predicting their geophysical properties and behaviour under core conditions. We will contrast what is known from experiment or approximate theory, with the developing insight which is coming from computational mineral physics research. Although interatomic potentials have been 
used to study Fe (e.g. Matsui and Anderson, 1997), many of its properties are very dependent upon a precise description of its metallic nature and can only be modelled accurately by quantum mechanical methods. Thus, below we briefly introduce the essential ab initio techniques used in the most recent studies of Fe alloys (see also Stixrude et al., 1998; Vočadlo et al., 2003; SteinleNeumann et al., 2003). We then present a discussion of the structure of the stable phase of $\mathrm{Fe}$ at core pressures and temperatures, its melting behaviour at core pressures, ab initio derived estimates of the composition of the core and its predicted thermal structure. We conclude with a materials-based discussion of the interpretation of the seismic structure of the inner core, and high-light some of the issues which must be addressed in the future.

\section{Quantum Mechanical Simulations}

To investigate the microscopic properties of matter from first principles one needs to solve the Schrödinger equation of the system:

$$
H \Psi=E \Psi
$$

where $H$ is the Hamiltonian, and is given by $H=T_{i}+T_{e}+V_{i i}+V_{i e}+V_{e e}$, with $T_{i}$ and $T_{e}$ representing the kinetic energies of the nuclei and electrons respectively, $V_{i i}$ the repulsive Coulombic energy between the nuclei, $V_{i e}$ the attractive Coulombic energy between the nuclei and the electrons and $V_{e e}$ the repulsive Coulombic energy between electrons, and $\Psi$ is the many body wave function of the system that depends on the positions of all the nuclei and electrons. Since the electrons are much less massive than the nuclei, their motion adiabatically follows that of the nuclei, and it is a reasonable approximation to decouple the electronic degrees of freedom from the ionic ones. Therefore, for each ionic configuration, $\mathbf{R}$, Eq. 1 can be written as:

$$
H(\{\mathbf{R}\}) \Psi=E(\{\mathbf{R}\}) \Psi
$$

where now $H(\{\mathbf{R}\})$ depends only parametrically on the position of the ions, $\mathbf{R}$, and one has to solve Eq. 2 only with respect to the electronic degrees of freedom. The resulting energy, $E(\{\mathbf{R}\})$, can then be used as the potential energy for the motion of the nuclei. Energy minimisation techniques may then be applied in order to obtain the equilibrium structure for the system under consideration.

Unfortunately, the complexity of the wave function, $\Psi$, for an $N$ electron system scales as $M^{N}$, where $\mathrm{M}$ is the number of degrees of freedom for a single electron wave function, $\psi$. This type of problem cannot readily be solved for large systems due to computational limitations, and therefore the exact solution to the problem for large systems is intractable. However, there are a number of approximations that may be made to simplify the calculation, whereby good predictions of the structural and electronic properties of materials can be obtained by solving self-consistently the one-electron Schrödinger equation for the system, and then summing these individual contributions over all the electrons in the system (for more detailed reviews see Gillan (1997) and Stixrude et al. (1998)). Fe and its alloys have been studied extensively by a number of groups (e.g., see Vočadlo et al., 2003; Steinle-Neumann et al., 2003), and it now appears that $a b$ initio calculations give an accurate description of the known properties of Fe. The calculations performed by us and others (e.g. Steinle-Neumann et al., 2003) are based on 
Density Functional Theory (DFT), generally within the Generalised Gradient Approximation (GGA). Our calculations have been performed using the VASP code (Kresse and Furthmüller, 1996), and most recently we have used the Projected-Augmented Wave (PAW) method (Blöchl, 1994) to calculate the total energy of the system. The PAW method is closely related to the ultra-soft pseudopotential method and has been shown to give results that agree accurately with all-electron methods (Kresse and Joubert, 1999; Alfè et al., 2000, 2001).

To study Fe under core conditions, however, we need not only to explore the energetics of bonding, but we are also concerned with the effect of temperature on the system. This requires us to calculate the Gibbs free energy of the systems, which can be done either using lattice dynamic or molecular dynamic methods. Lattice dynamics is a semi-classical approach that can be used with the quasiharmonic approximation (QHA) to describe a cell in terms of independent quantised harmonic oscillators, the frequencies of which vary with cell volume, thus allowing for a description of thermal expansion (e.g. Born and Huang, 1954). The motions of the individual particles are treated collectively as lattice vibrations or phonons, and the phonon frequencies, $\omega(\mathbf{q})$, and displacement eigenvectors, $\mathbf{e}(\mathbf{q})$, are obtained by solving:

$$
m \omega^{2}(\mathbf{q}) \mathbf{e}_{\mathrm{i}}(\mathbf{q})=\mathbf{D}(\mathbf{q}) \mathbf{e}_{\mathrm{j}}(\mathbf{q})
$$

where $m$ is the mass of the atom, and the dynamical matrix, $\mathbf{D}(\mathrm{q})$, is defined by:

$$
\mathbf{D}(\mathbf{q})=\sum_{\mathrm{ij}}\left(\frac{\partial^{2} \mathrm{U}}{\partial \mathrm{u}_{\mathrm{i}} \partial \mathrm{u}_{\mathrm{j}}}\right) \exp \left(\mathrm{iq} \cdot \mathbf{r}_{\mathrm{ij}}\right)
$$

where $U$ is the potential energy of the system, $\mathbf{r}_{\mathrm{ij}}$ is the interatomic separation, and $\mathrm{u}_{\mathrm{i}}$ and $\mathrm{u}_{\mathrm{j}}$ are the displacements of atoms $i$ and $j$ from their equilibrium position. For a unit cell containing $\mathrm{N}$ atoms, there are $3 \mathrm{~N}$ eigenvalue solutions $\left(\omega^{2}(\mathbf{q})\right)$ for a given wave vector, $\mathbf{q}$. There are also $3 \mathrm{~N}$ sets of eigenvectors $\left(\mathbf{e}_{\mathbf{x}}(\mathbf{q}), \mathbf{e}_{\mathbf{y}}(\mathbf{q}), \mathbf{e}_{\mathbf{z}}(\mathbf{q})\right)$ which describe the pattern of atomic displacements for each normal mode.

The vibrational frequencies of a lattice can be calculated $a b$ initio, by standard methods such as the small-displacement method (e.g. Kresse et al., 1995). Having calculated the vibrational frequencies, a number of thermodynamic properties may then be calculated using standard statistical mechanical relations, which are direct functions of these vibrational frequencies. Thus for example the Helmholtz free energy, $F$, is given by:

$$
F=k_{B} T \sum_{i}^{M}\left(\frac{x_{i}}{2}+\ln \left(1-e^{-x_{i}}\right)\right)
$$

where $\mathrm{x}_{\mathrm{i}}=h \omega_{\mathrm{i}} / 2 \pi k_{B} T, h$ is Planck's constant and $k_{B}$ is Boltzmann's constant, and the sum is over all the $\mathrm{M}=3 \mathrm{~N}$ normal modes. Modelling the effect of pressure is essential if one is to obtain accurate predictions of phenomena such as phase transformations and anisotropic compression. This problem is now routinely being solved using codes that allow constant stress, variable geometry cells in both static and dynamic simulations. In the case of lattice dynamics, the mechanical pressure is calculated from strain derivatives, whilst the thermal kinetic pressure is calculated from phonon frequencies (e.g. Parker and Price, 1989). The balance of these forces can be used to determine the variation of cell size as a function of pressure and temperature. 
Molecular dynamics is routinely used for medium to high temperature simulations of minerals and in all simulations of liquids, where lattice dynamics is of course inapplicable. The method is essentially classical, and its details are presented in, for example, Allen and Tildesley (1987). The interactions between the atoms within the system have traditionally been described in terms of the interatomic potential models mentioned earlier, but instead of treating the atomic motions in terms of lattice vibrations, each ion is treated individually. As the system evolves, the required dynamic properties are calculated iteratively at the specified pressure and temperature. The ions are initially assigned positions and velocities within the simulation box; their co-ordinates are usually chosen to be at the crystallographically determined sites, whilst their velocities are equilibrated such that they are compatible with the required system temperature, and such that both energy and momentum is conserved. In order to calculate subsequent positions and velocities, the forces acting on any individual ion are then calculated from the first derivative of the total energy function, and the new position and velocity of each ion may be calculated at each time-step by solving Newton's equation of motion. Both the particle positions and the volume of the system, or simulation box, can be used as dynamical variables, as is described in detail in Parrinello and Rahman (1980) and by Wentzcovitch (1991). The kinetic energy, and therefore temperature, is obtained directly from the velocities of the individual particles. With this explicit particle motion, the anharmonicity is implicitly accounted for at high temperatures.

Because of advances in computer power, it is now possible to perform ab initio molecular dynamics (AIMD), with the forces calculated fully quantum mechanically instead of relying upon the use of interatomic potentials (see for example Scandolo, 2002). The first pioneering work in AIMD was that of Car and Parrinello (1985), who proposed a unified scheme to calculate $a b$ initio forces on the ions and keep the electrons close to the Born-Oppenheimer surface while the atoms move. We have used in the work summarized below an alternative approach, in which the dynamics are performed by explicitly minimizing the electronic free energy functional at each time step. This minimization is more expensive than a single CarParrinello step, but the cost of the step is compensated by the possibility of making longer time steps.

\section{The Properties of Fe under Core Conditions}

\section{The High P Structure of Fe}

Before we can even begin to provide a materials based interpretation of the composition and structure of the core, we must understand the behaviour of its primary constituent $(\mathrm{Fe})$ under core conditions. At ambient conditions, Fe is crystalline and has a body centred cubic (bcc) structure. This transforms with temperature to a face centred cubic $(f c c)$ form, and with pressure transforms to a hexagonal close packed ( $h c p$ ) phase, $\varepsilon$-Fe. The high $P / T$ phase diagram of pure iron itself however is still controversial (see Figure 1 and also the discussions in Stixrude and Brown (1998), Anderson (2003), and Steinle-Neumann et al., (2003)). Various diamond anvil cell (DAC) based studies have been interpreted as showing that hcp Fe transforms at high temperatures to a phase which has variously been described as having a double hexagonal close packed structure $(d h c p)$ (Saxena et al., 1996) or an orthorhombicly distorted hcp structure (Andrault et al., 1997). Furthermore, high pressure shock experiments have also been interpreted as showing a high pressure solid-solid phase transformation (Brown and McQueen, 
1986; Brown, 2001), which has been suggested could be due to the development of a $b c c$ phase (Matsui and Anderson, 1997). Other experimentalists, however, have failed to detect such a post-hcp phase (e.g. Shen et al., 1998; Nguyen and Holmes, 1999), and have suggested that the previous observations were due either to minor impurities or to metastable, strain-induced behaviour.

Further progress in interpreting the nature and evolution of the core would be severely hindered if the uncertainty concerning the crystal structure of the core's major chemical component remained unresolved. An alternative approach to this problem, however, is to use $a b$ initio calculations, which have been shown to provide an accurate means of calculating the properties of materials at high $P$ and $T$ (e.g. Gillan, 1997). Thus, Vočadlo et al., 1999 carried out a series of calculations to determine ab initio the stable phase of Fe. Here, we performed spin polarized simulations on candidate phases (including a variety of distorted $b c c$ and $h c p$ structures and the $d h c p$ phase) at pressures ranging from 325 to $360 \mathrm{GPa}$. These revealed, in agreement with Söderlind et al. (1996), that at core pressures only $b c c$ Fe has a residual magnetic moment and all other phases have zero magnetic moments. We found that at core pressures and zero temperature, both the $b c c$ and the suggested orthorhombic polymorph of iron (Andrault et al., 1997) are mechanically unstable. The $b c c$ phase can be continuously transformed to the $f c c$ phase (confirming the findings of Stixrude and Cohen, 1995), while the orthorhombic phase spontaneously transforms to the $h c p$ phase, when allowed to relax to a state of isotropic stress. In contrast, $h c p$, $d h c p$ and $f c c$ Fe remain mechanically stable at core pressures, and we were therefore able to calculate their phonon frequencies and free energies. It was concluded that, on the basis of lattice dynamic calculations over the whole P-T space investigated, the hcp phase of Fe has the lowest Gibbs free energy, and is therefore the stable form of Fe under core conditions, indicating that the true phase diagram for $\mathrm{Fe}$ is simpler than previously suggested (Fig. 1) and is better described by Fig. 2 .

\section{The High P Melting of Fe}

Having shown how ab initio calculations can be used to establish the sub-solidus phase relations in high $\mathrm{P} \mathrm{Fe}$, we now consider its high $P / T$ melting behaviour. The temperature distribution in the core is poorly constrained and consequently a reliable estimate of the melting temperature of $\mathrm{Fe}$ at the pressure of the inner-core boundary (ICB) would be valuable. There is much controversy over the high $\mathrm{P}$ melting behaviour of $\mathrm{Fe}$ (e.g. see Shen and Heinz, 1998), with estimates of the $T_{m}$ of Fe at ICB pressures ranging between $\sim 4500 \mathrm{~K}$ to $\sim 7500 \mathrm{~K}$.

Since both our calculations and recent experiments (Shen et al., 1998) suggest that Fe melts from the $\varepsilon$-phase in the pressure range immediately above $60 \mathrm{GPa}$, we focus here on the equilibrium between hcp $\mathrm{Fe}$ and liquid $\mathrm{Fe}$. The condition for two phases to be in thermodynamic equilibrium at a given temperature and pressure is that their Gibbs free energies, $G(P, T)$, are equal. To determine $T_{m}$ at any pressure, we calculate $G$ for the solid and liquid phases as a function of $T$ and determine where they are equal. In fact, we calculate the Helmholtz free energy, $F(V, T)$, as a function of volume, $V$, and hence obtain the pressure through the relation $P=-(\partial F / \partial V)_{T}$ and $G$ through its definition, $G=F+P V$. 
To validate our method and to prove its accuracy, we modeled the well studied high $P$ melting behaviour of Al (de Wijs et al., 1998; Vočadlo and Alfè, 2002). Figure 3 shows the very good agreement obtained for this system. Our model for $T_{m}$ of Fe is shown in Fig. 4, and we conclude that Fe melts at ICB pressures between 6,200 and 6,350 K (Alfè et al., 1999, $2002 \mathrm{~b}, \mathrm{~d})$. A full analysis of the errors and uncertainties in these calculations has been reported in Alfè et al. (2002b,d). For pressures $P<200 \mathrm{GPa}$ (the range covered by DAC experiments) the $a b$ initio curve lies $\sim 900 \mathrm{~K}$ above the experimental values of Boehler (1993) and $\sim 200 \mathrm{~K}$ above the more recent values of Shen et al. (1998) (who stress that their values are only a lower bound to $T_{m}$ ). The ab initio curve falls significantly below the shock-based estimates for the $T_{m}$ of Yoo et al. (1993), in whose experiments temperature was deduced by measuring optical emission (however, the difficulties of obtaining temperature by this method in shock experiments are well known), but accords almost exactly with the shock data values of Brown and McQueen (1986) and Nguyen and Holmes (1999).

There are other ways of determining the melting temperature of a system by $a b$ initio methods, including performing simulations that model co-existing liquid and crystal phases. The melting temperature of such a system can then be inferred by seeing which of the two phases grows during the course of a series of simulations at different temperatures. This intuitively attractive approach was used by Laio et al. (2000) and by Belonoshko et al. (2000) to study the melting of Fe. But because of current computer limitations, they had to modelled Fe melting using interatomic potentials fitted to ab initio surfaces. We have recently also used the co-existence method (Alfè et al., 2002d), but with a model potential fitted to our own ab initio calculations. Initially, our raw model failed to give the same melting temperature as obtained from our ab initio free energy cacultions, but when the results were corrected for the free energy mismatch of the model potential with respect to the $a b$ initio energies of liquid and solid, the results for the two methods came into agreement. Thus the shortcomings of model potential co-existence calculations can be corrected by calculating the free energy differences between the model and the $a b$ initio system for both the liquid and solid phases. This difference in free energy between liquid and solid can then be transformed into an effective temperature correction. When this is done to Belonoshko's data, there is excellent agreement with our ab initio melting curve for Fe (see Fig. 4). In the future, when greater computing resources will allow full ab initio simulations, the co-existence approach may prove the more attractive strategy for modelling melting behaviour, as it is an intuitive simulation of the melting process.

Interestingly Poirier and Shankland (1993) obtained a value of $T_{m}$ for Fe at $330 \mathrm{GPa}$ of 6100 $\pm 100 \mathrm{~K}$ by using the more empirical dislocation melting model, and Anderson (2003) finds a $T_{m}$ of $5900 \pm 300 \mathrm{~K}$ on the basis of thermodynamic analysis. Thus with our ab initio calculated value of 6200 to $6350 \mathrm{~K}$ having been shown to be robust, there appears to be now an emerging consensus on the high $P$ melting of pure Fe. However to understand the core, we also need to investigate the effect of other alloying elements on the melting behaviour of $\mathrm{Fe}$. Some of these issues are address below.

\section{The Composition and Temperature of the Core}


Seismological data suggest that the outer and inner core contain some light element impurities (Birch, 1964). Cosmochemical abundances of the elements, combined with models of the Earth's history, limit the possible impurities to a few candidates. Those most often discussed are S, O and Si (e.g. Poirier, 1994b; Allègre et al., 1995; McDonough and Sun, 1995), and we have to date confined our studies to these three. Our strategy for constraining the impurity fractions and the temperature of the core is based on the supposition that the solid inner core is slowly crystallising from the liquid outer core, and that therefore the inner and outer core are in thermodynamic equilibrium at the ICB. This implies that the chemical potentials of $\mathrm{Fe}$ and of each impurity must be equal on the two sides of the ICB.

If the core consisted of pure Fe, equality of the chemical potential (Gibbs free energy in this case) would tell us only that the temperature at the ICB is equal to the melting temperature of Fe at the ICB pressure of $330 \mathrm{GPa}$. With impurities present, equality of the chemical potentials for each impurity element imposes a relation between the mole fractions in the liquid and the solid, so that with $\mathrm{S}, \mathrm{O}$ and $\mathrm{Si}$ we have three such relations. But these three relations must be consistent with the accurate values of the mass densities in the inner and outer core deduced from seismic and free-oscillation data. Below we outline our finding (Alfè et al., 2002c) that ab initio results for the densities and chemical potentials in the liquid and solid $\mathrm{Fe} / \mathrm{S}, \mathrm{Fe} / \mathrm{O}$ and $\mathrm{Fe} / \mathrm{Si}$ alloys determine with useful accuracy the mole fraction of $\mathrm{O}$ and the sum of the $\mathrm{S}$ and $\mathrm{Si}$ mole fractions in the outer and inner core, as well as enabling us to determine the temperature at the ICB.

The chemical potential, $\mu_{x}$, of a solute $x$ in a solid or liquid solution is conventionally expressed as $\mu_{x}=\mu_{x}{ }^{0}+k_{B} \operatorname{Tln} a_{x}$, where $\mu_{x}^{0}$ is a constant and $a_{x}$ is the activity. It is common practice to write $a_{x}=\gamma_{x} c_{x}$, where $\gamma_{x}$ is the activity coefficient and $c_{x}$ the concentration of $x$. The chemical potential can therefore be expressed as:

which we rewrite as:

$$
\mu_{x}=\mu_{x}^{0}+k_{B} T \ln \gamma_{x} c_{x}
$$

$$
\mu_{x}=\mu_{x}^{*}+k_{B} T \ln c_{x}
$$

It is helpful to focus on the quantity $\mu_{x}{ }^{*}$ for two reasons: first, because it is a convenient quantity to obtain by ab initio calculations (Alfè et al., 2002c); second, because at low concentrations the activity coefficient, $\gamma_{x}$, will deviate only weakly from unity by an amount proportional to $c_{x}$, and by the properties of the logarithm the same will be true of $\mu_{x}{ }^{*}$.

The equality of the chemical potentials $\mu_{x}^{l}$ and $\mu_{x}^{s}$ in coexisting liquid and solid (superscripts 1 and $s$ respectively) then requires that:

or equivalently

$$
\mu_{x}^{* l}+k_{B} \operatorname{Tln} c_{x}^{l}=\mu_{x}^{{ }^{*}}+k_{B} \operatorname{Tln} c_{x}^{s}
$$

$$
c_{x}^{s} / c_{x}^{l}=\exp \left[\left(\mu_{x}^{* l}-\mu_{x}^{*}\right) / k_{B} T\right]
$$

This means that the ratio of the mole fractions $c_{x}^{s}$ and $c_{x}^{l}$ in the solid and liquid solution is determined by the liquid and solid thermodynamic quantities $\mu_{x}{ }^{*}$ and $\mu_{x}{ }^{*_{s}}$. Although liquidsolid equilibrium in the $\mathrm{Fe} / \mathrm{S}$ and $\mathrm{Fe} / \mathrm{O}$ systems has been experimentally studied up to 
pressures of around $60 \mathrm{GPa}$ (e.g Boehler, 2000), there seems little prospect of obtaining experimental data for $\mu_{x}{ }^{*}-\mu_{x}{ }^{*}$ for Fe alloys at the much higher ICB pressure. However, we have shown (Alfè et al., 2002c) recently that the fully ab initio calculation of $\mu_{x}{ }^{* l}$ and $\mu_{x}{ }^{*_{s}}$ is technically feasible. Thus, the chemical potential, $\mu_{x}$, of chemical component $x$ can be defined as the change of Helmholtz free energy when one atom of $x$ is introduced into the system at constant temperature, $T$, and volume, $V$. In ab initio simulations, it is awkward to introduce a new atom, but the awkwardness can be avoided by calculating $\mu_{x}{ }^{*}-\mu_{F e}{ }^{*}$, which is the free energy change, $\Delta F$, when an $\mathrm{Fe}$ atom is replaced by an $x$ atom. For the liquid, this $\Delta F$ is computed by applying the technique of 'thermodynamic integration' to the (hypothetical) process in which an Fe atom is continuously transmuted into an $x$ atom. We have recently performed such calculations for transmuting Fe atoms into S, O and Si (Alfè et al., 2002a).

Alfè et al. $(2000,2002 \mathrm{c})$ performed simulations at constant volume and temperature on systems of 64 atoms; the duration of the simulations after equilibration was typically 6 ps in order to reduce statistical errors to an acceptable level; the number of thermodynamic integration points used in transmuting $\mathrm{Fe}$ into $x$ was 3 , and we carefully checked the adequacy of these numbers of points. Our results reveal a major qualitative difference between $\mathrm{O}$ and the other two impurities. For $\mathrm{S}$ and $\mathrm{Si}, \mu_{x}{ }^{*}$ is almost the same in the solid and the liquid, the differences being at most $0.3 \mathrm{eV}$, i.e. markedly smaller than $k_{B} T \sim 0.5 \mathrm{eV}$; but for $\mathrm{O}$ the difference of $\mu_{x}{ }^{*}$ between solid and liquid is $\sim 2.6 \mathrm{eV}$, which is much bigger than $k_{B} T$. This means that added $\mathrm{O}$ will partition strongly into the liquid, but added $\mathrm{S}$ or $\mathrm{Si}$ will have similar concentrations in the two phases.

Our simulations of the chemical potentials of the alloys can be combined with simulations of their densities to investigate whether the known densities of the liquid and solid core can be matched by any binary $\mathrm{Fe} / x$ system, with $x=\mathrm{S}, \mathrm{O}$ or $\mathrm{Si}$. Using our calculated partial volumes of $\mathrm{S}, \mathrm{Si}$ and $\mathrm{O}$ in the binary liquid alloys, we find that the mole fractions required to reproduce the liquid core density are 16,14 and $18 \%$ respectively (Fig. 5, panel (a) displays our predicted liquid density as a function of $\mathrm{c}_{\mathrm{x}}$ compared with the seismic density). Our calculated chemical potentials in the binary liquid and solid alloys then give the mole fractions in the solid of 14,14 and $0.2 \%$ respectively that would be in equilibrium with these liquids (see Fig. 5, panel (b)). Finally, our partial volumes in the binary solids give ICB density discontinuities of $2.7 \pm 0.5,1.8 \pm 0.5$ and $7.8 \pm 0.2 \%$ respectively (Fig. 5, panel (c)). As expected, for $\mathrm{S}$ and $\mathrm{Si}$, the discontinuities are considerably smaller than the known value of $4.5 \pm 0.5 \%$; for $\mathrm{O}$, the discontinuity is markedly greater than the known value. We conclude that none of the binary systems can account for the discontinuity quantitatively. However, it clearly can be accounted for by $\mathrm{O}$ together with either or both of $\mathrm{S}$ and $\mathrm{Si}$. Ab initio calculations on general quaternary alloys containing $\mathrm{Fe}, \mathrm{S}, \mathrm{O}$ and $\mathrm{Si}$ will be feasible in the future, but currently they are computationally too demanding, so for the moment we assume that the chemical potential of each impurity species is unaffected by the presence of the others. Our estimated mole fractions needed to account for the ICB density discontinuity, were reported in Alfè et al. (2002a) as being 8.5 2.5 mole \% S/Si and $0.2 \pm 0.1 \% \mathrm{O}$ in the inner core and $10 \pm 2.5 \% \mathrm{~S} / \mathrm{Si}$ and $8 \pm 2.5 \% \mathrm{O}$ in the liquid outer core. This compositional estimate was based on the value of the density discontinuity at the ICB determined by Shearer and Masters (1990). Since then, Masters and Gubbins (2003) have reassessed the free oscillation data set, and have determined the density jump at the ICB to be $0.82 \pm 0.18 \mathrm{Mg} \mathrm{m}^{-3}$, which is larger than the previous estimates. Using the new density data of Masters and 
Gubbins (2003) leads to a revised core composition of $7 \pm 2.5$ mole $\% \mathrm{~S} / \mathrm{Si}$ and $0.2 \pm 0.1 \% \mathrm{O}$ for the inner core and $8 \pm 2.5 \% \mathrm{~S} / \mathrm{Si}$ and $13 \pm 2.5 \% \mathrm{O}$ for the outer core. This change in our estimate of core composition emphasises the need for very accurate seismic data.

With the calculated impurity chemical potentials, we can use the Gibbs-Duhem relation to compute the change in the Fe chemical potential caused by the impurities in the solid and liquid phases (Alfè et al., 2002a). By requiring the chemical potential of $\mathrm{Fe}$ to be the same in both phases we can calculate the liquidus temperature in the iron alloy system. For our estimated liquid compositions, we calculate that the liquidus temperature of the core alloy is between 700 and $800 \mathrm{~K}$ lower than the melting temperature of pure $\mathrm{Fe}$, depending upon the value of the density contrast at the ICB. Thus we calculate that the Earth's temperature at the ICB to be between 5400 and $5650 \mathrm{~K}$.

Using this calculated value for the ICB temperature, and our $a b$ initio values for the Grüneisen parameter, $\gamma$, for liquid Fe along the outer core adiabat (Vočadlo et al., 2003), it is possible to determine the core temperature at the $\mathrm{CMB}$ from the relation defining the adiabatic temperature gradient:

$$
\partial T / \partial r=-\gamma g T / \Phi
$$

where $\Phi$ is the seismic parameter and $g$ is the acceleration due to gravity. Like Anderson (2003), we find $\gamma$ to be $\sim 1.5$ and virtually constant in the outer core, which leads to an $a b$ initio estimate of the core temperature at the CMB of between 3950 and $4200 \mathrm{~K}$. Again, this range is in excellent agreement with that inferred from thermodynamic arguments (Anderson, 2003).

\section{Possible Structure of the Inner Core}

The conventional interpretation of the origin of the seismic anisotropy of the inner core is based on the idea of the development of partial alignment of the elastically anisotropic crystals of $h c p$ Fe (e.g. Song, 1997). The static elastic constant of $h c p$ Fe were first calculated by Stixrude and Cohen (1995) and then by Söderlind et al. (1996). The low temperature elastic constants of $h c p$ Fe at 39 and $211 \mathrm{GPa}$ were measured in an experiment reported by Mao et al. (1999). The overall agreement between the experimental and various ab initio studies is very good, and the measured and calculated bulk and shear moduli and the seismic velocities of $h c p \mathrm{Fe}$ as a function of pressure are shown in Figs 6 and 7. The calculated values compare well with experimental data at higher pressures, but discrepancies at lower pressures are probably due to the neglect of magnetic effects in the simulations (see also the discussion in Steinle-Neumann et al., 2003).

The effect of temperature on the elastic constants of Fe was reported by Steinle-Neumann et al. (2001) based on calculations using the approximate "particle in a cell" method. With increasing temperature, they found a significant change in the $c / a$ axial ratio of the $h c p$ structure, which in turn caused a marked reduction in the elastic constants $c_{33}, c_{44}$ and $c_{66}$. This led them to conclude that increasing temperature reverses the sense of the single crystal longitudinal anisotropy of $h c p \mathrm{Fe}$, and that the anisotropy of the core should now be viewed as being due to $h c p \mathrm{Fe}$ crystals having their c-axis preferably aligned equatorially, rather than 
axially as originally suggested by Stixrude and Cohen (1995). However, the $a b$ initio determination of high $T$ elastic constants is very difficult. Karki et al (1999) and Oganov et al. (2001) have shown how to use truly ab initio methods to obtain high $T$ elastic constants, and further work is needed to confirm the high $T$ properties of $h c p$ Fe. Recent calculations (Gannarelli et al., 2003) have failed thus far to reproduce the strong effect of temperature on $c / a$ seen by Steinle-Neumann et al., and if this result is confirmed by more precise molecular dynamic simulations, then it will have important implications for the interpretation of the seismic tomography of the inner core.

The nature of inner core anisotropy has recently been shown to be more complex than previously thought, and Beghein and Trampert (2003) have shown that free oscillation data cannot be simply interpreted in terms of the elastic properties of $h c p$ Fe as reported by Steinle-Neumann et al. (2001). Furthermore, the assumption that hcp Fe is the thermodynamically stable polymorph of $\mathrm{Fe}$ at the high temperatures found in the inner core has also been recently questioned (Brown, 2001). In spite of the arguments in favour of hcp $\mathrm{Fe}$, it has been proposed by a number of workers that $b c c$ Fe might be the stable high $P / T$ phase (Ross et al, 1990; Matsui and Anderson, 1997). A strong argument in favour of the stability of $b c c$ Fe under these conditions is that a number of transition metals (e.g. Ti, Zr..) are known to transform from close-packed structures to the $b c c$ structure at temperatures just below their melting curve. However, until recently it appeared that theoretical calculations had ruled out the $b c c$ structure as a candidate for the stable phase of Fe in the core. As reviewed in for example Steinle-Neumann et al. (2003) this was because ab initio calculations have shown that the $b c c$ structure becomes elastically unstable at pressures above $\sim 150 \mathrm{GPa}$, and that the enthalpy of the perfect $b c c$ structure is considerably higher than that of the hcp phase. However, these arguments are not conclusive because they are based on athermal or lattice dynamical $a b$ initio calculations, which because of the dynamical instability of $b c c$ Fe cannot be used to determine entropic effects at high pressures. In the past, computing limitations prevented a more sophisticated analysis, but recent methodological developments mean that it is now possible accurately to address these thermal effects. Thus, to resolve the controversy over the effect of temperature on the stability of $b c c \mathrm{Fe}$, we recently performed $a b$ initio molecular dynamics (AIMD) calculations on $b c c$ Fe to simulate directly its behaviour at the high temperatures relevant to the Earth's inner core (Vočadlo et al, 2003).

Table 1 shows the calculated Helmholtz free energies for the $b c c$ and $h c p$ phases over a range of volumes and temperatures along (and below) the previously determined melting curve of $h c p \mathrm{Fe}$. It is clear that in all cases $F_{b c c}>F_{h c p}$, and so as previously concluded (on the basis of lattice dynamical as opposed to molecular dynamical calculations) pure Fe adopts the $h c p$ structure at core pressures and temperatures. However, the differences in free energies are small (33-58 meV/atom along $T_{m}$ ). The Earth's inner core is known not to be made of pure $\mathrm{Fe}$, but is expected to be alloyed with between 5 to $10 \mathrm{~mol} \%$ of a lighter element. It is argued that, either separately or together, $\mathrm{S}$ and $\mathrm{Si}$ are two of the most probable light elements alloyed with Fe in the core (Poirier, 1994a; Allègre et al., 1995). Recent experiments (Dobson et al., 2002) have shown that at high pressures FeSi crystallises with the CsCl-structure (i.e. has identical atomic co-ordinates to $b c c-\mathrm{Fe}$ ), and it has been found that at low concentrations Si favours the formation of $b c c$ Fe over the $h c p$ polymorph (Lin et al., 2002; Dubrovinsky et al., 2003). We therefore investigated the energetic effect of the substitution of S and Si in $b c c$ 
and $h c p \mathrm{Fe}$ at representative core densities. We found that the enthalpies of the $\mathrm{S}$ and $\mathrm{Si}$ defects are respectively 1.4 and $1.2 \mathrm{eV}$ per defect atom more stable in the $b c c$ structure than in the $h c p$ phase. Therefore, for example, a $5 \mathrm{~mol} \%$ concentration of $\mathrm{Si}$ in $\mathrm{Fe}$, would stabilise the $b c c$ phase by $60 \mathrm{meV}$. Thus, we conclude that the presence of S or Si as the light impurity element in the core, at appropriate concentrations, could favour the formation of a $b c c$ - rather than an $h c p$-structured $\mathrm{Fe}$ alloy phase at temperatures just below $T_{m}$ at inner core pressure. The presence of a $b c c$-structured alloy may, therefore, be a candidate for explaining the observed seismic complexity of the inner core recently reported by Beghein and Trampert (2003), however more work on the high $T$ elasticity of this and other candidate phases is needed before the seismic anisotropy of the inner core can be fully understood.

\section{Conclusion}

The past decade has seen a major advance in the application of ab initio methods in the solution of high pressure and temperature geophysical problems, thanks to the rapid developments in high performance computing. We are now in a position to calculate from first principles the free energies of solid and liquid phases, and hence to determine both the phase relations and the physical properties of planetary forming phases. So far we have been able to study the effects of $\mathrm{S}, \mathrm{O}$ and $\mathrm{Si}$ on the behaviour of $\mathrm{Fe}$, but in the future it will be essential also to consider other elements, including $\mathrm{Ni}, \mathrm{C}, \mathrm{H}$ and $\mathrm{K}$. However, from what has been studied to date, it is likely that the ICB temperature lies between 5400 and $5650 \mathrm{~K}$ and the core temperature at the CMB is between 3950 and $4200 \mathrm{~K}$. Using ab initio methods it has been shown the composition of the core can be constrained, but estimates are very sensitive to the seismologically inferred values of core density.

In the future, the clearer insight that ab initio modelling gives to our understanding of the properties of the core, will enable the development of more quantitative descriptions of the history of the Earth, and even to the establishment of quantitative models of the geodynamo (see for example Gubbins et al., 2003). The high $P$ and $T$ structure and elastic properties of Fe and its alloys are still the subject of some uncertainty, but theory should soon be able to resolve these issues.

We look forward to the advent of routinely available 'terascale' computing. This will open new possibilities for geophysical modelling. It will be possible to model more complex and larger systems, to investigate for example solid state rheological problems, or physical properties such as thermal and electrical conductivity, and to model the possible chemical processes occurring at the CMB. However, it must be recognised that the DFT methods, that are currently used, are still approximate, and fail for example to describe the band structure of important phases such as $\mathrm{FeO}$. In the future it will be necessary to consider using terascale facilities to implement more demanding but more accurate techniques, such as those based on quantum Monte Carlo methods. Nevertheless, significant progress has been made to date, and it is certain than in the future further insights from computational mineral physics will enhance the understanding of our planet.

\section{Acknowledgements}

DA and LV are supported by Royal Society University Research Fellowships. DA also 
acknowledges support from the Leverhulme Trust. MJG thanks GEC and Daresbury Laboratory for their support. Our work reported in this paper was supported by NERC grants GR3/12083 and GR9/03550. The calculations were run on the Cray T3D and Cray T3E machines at Edinburgh and the Manchester CSAR Centre, and on the Origin 2000 machine at the UCL HiPerSPACE Centre. We would like to thank Renata Wentzcovitch, Steve Sparks and Sandro Scandolo for helpful comments and corrections. 


\section{References}

Alfè, D., 1998. Program available from, http://chianti.geol.ucl.ac.uk/ dario/phon.tar.z

Alfè, D., Gillan, M.J., and Price, G.D., 1999. The melting curve of iron at the pressures of the Earth's core from $a b$ initio calculations. Nature, 401: 462-464.

Alfè, D., Gillan, M.J., and Price, G.D., 2002a. Composition and temperature of the Earth's core constrained by combining ab initio calculations and seismic data. Earth Planet. Sci. Lett., 195: 91-98.

Alfè, D., Gillan, M.J., and Price, G.D., 2002c. Ab initio chemical potential of solid and liquid solutions and chemistry of the Earth's core. J. Chemical Physics. 116: 7127-7136.

Alfè, D., Gillan, M.J., and Price, G.D., 2002d. Complementary approaches to the $a b$ initio calculation of melting properties. J. Chemical Physics. 116: 6170-6177.

Alfè, D., Price, G.D., and Gillan, M.J., 2001. Thermodynamics of hexagonal-close-packed iron under Earth's core conditions. Physical Review. B64: 045123.

Alfè, D., Price, G.D., and Gillan, M.J., 2002b. Iron under Earth's core conditions: Liquidstate thermodynamics and high-pressure melting curve from ab initio calculations. Phys. Review. B65: 165118, 1-11.

Allègre, C.J., Poirier, J.P., Humler, E., and Hofmann, A.W., 1995. The chemical composition of the Earth. Phys. Earth Planet. Interiors. 134: 515-526.

Allen, M.P., and Tildesley, D.J., 1987. Computer Simulation of Liquids. Oxford University Press, Oxford, UK.

Anderson, O.L., 1995. Equations of state of solids for Geophysics and ceramic science. Oxford University Press, Oxford, UK.

Anderson, O.L., 2003. The three-dimensional phase diagram of iron. In: Earth's Core (eds. V. Dehant, K.C. Creager, S-i. Karato, S. Zatman). AGU, Geodynamics Series, 31, 83-104.

Andrault, D., Fiquet, G., Kunz, M., Visocekas, F., and Häusermann, D., 1997. The orthorhombic structure of iron: an in situ study at high temperature and high pressure. Science. 278: 831-834.

Beghein, C., and Trampert, J., 2003. Robust Normal Mode Constraints on Inner-Core

Anisotropy from Model Space Search. Science, 299, 552-555.

Belonoshko, A.B., Ahuja, R., and Johansson, B., 2000. Quasi- ab initio molecular dynamic study of Fe melting. Phys Rev Lett. 84: 3638-3641.

Birch, F., 1952. Elasticity and the constitution of the Earth's interior. J Geophys. Res. 57: 227-286.

Birch, F., 1964. Density and composition of mantle and core. J Geophys Res, 69, 4377-4388.

Blöchl, P. E., 1994. Projector augmented-wave method. Phys. Rev. B 50, 17953-17979.

Boehler, R., 1993. Temperature in the Earth's core from the melting point measurements of iron at high static pressures. Nature. 363: 534-536.

Boehler, R., 2000. High-pressure experiments and the phase diagram of lower mantle and core materials. Reviews of Geophys. 38: 221-245.

Born, M., and Huang, K., 1954. Dynamical Theory of Crystal Lattices. Oxford University Press, Oxford, UK.

Brown, J.M., 2001. The equation of state of Iron to 450GPa: another high pressure solid phase? Geophys Res. Letts. 28: 4339-4342.

Brown, J.M., and McQueen, R.G., 1986. Phase transitions, Grüneisen parameter and elasticity for shocked iron between $77 \mathrm{GPa}$ and $400 \mathrm{GPa}$. J. Geophys. Res. 91: 7485-7494.

Car, R., and Parrinello, M., 1985. Unified Approach for Molecular Dynamics and Density 
Functional Theory. Phys. Rev. Lett. 55: 2471-2474.

Creager, K.C., 1992. Anisotropy of the inner core from differential travel times of the phases PKP and PKIKP. Nature, 356, 309-314.

Dobson, D.P., Vočadlo, L., and Wood, I.G., 2002. A new high-pressure phase of FeSi. Amer. Mineral. 87, 784-787.

Dubrovinsky, L., Dubrovinskaia, N., Langenhorst, F., Dobson, D., Rubie, D., Gessmann, C., Abrikosov, I.A., Johansson, B., Baykov, V.I., Vitos, L., Le Bihan, T., Crichton, W.A., Dmitriev, V., and Weber, H.P., 2003. Iron-silica interaction at extreme conditions and the electrically conducting layer at the base of Earth's mantle. Nature, 422, 58-61.

Dziewonski, A.M., and Gilbert, F., 1971. Solidity of the inner core of the Earth inferred from normal mode observations. Nature, 234, 465-466.

Fiquet, G., Badro, J., Guyot, F., Requardt, H., and Krisch, M., 2001. Sound velocities in iron to 110 gigapascals. Science, 291, 468-47.

Gannarelli, C.M.S., Alfè, D., and Gillan, M.J. (2003) The particle-in-cell model for ab initio thermodynamics: implications for the elastic anisotropy of the Earth's inner core. Phys. Earth Planet. Interiors, Submitted.

Gao, F., Johnston, R.L., and Murrell, J.N., 1993. Empirical many-body potential energy functions for Iron. J. Phys. Chem. 97: 12073-12082.

Gillan, M.J., 1997. The virtual matter laboratory. Contemp. Phys. 38: 115-130.

Gubbins, D., Alfè, D., Masters, G., Price, G.D., and Gillan, M.J., 2003. Can the Earth's dynamo run on heat alone? Geophys. J. Int., 155, 609-622.

Gutenberg, B., 1913. Uber die Konstitution der Erdinnern, erschlossen aus Erdbebenbeobachtungen. Phys. Zeit., 14, 1217

Ishii, M., and Dziewonski, A.M., 2002. The innermost inner core of the earth: Evidence for a change in anisotropic behaviour at the radius of about $300 \mathrm{~km}$. Proceedings of the National Academy Of Sciences Of The United States Of America . 99: 14026-14030.

Karki, B.B., Wentzcovitch, R.M., de Gironcoli, S., Baroni, S.,1999. First-principles determination of elastic anisotropy and wave velocities of $\mathrm{MgO}$ at lower mantle conditions. Science, 286: 1705-1707.

Kresse, G., Furthmüller, J., and Hafner, J., 1995. Ab-initio force-constant approach to phonon dispersion relations of diamond and graphite. Europhys. Lett. 32: 729-734.

Kresse, G., and Furthmüller, J., 1996. Efficient iterative schemes for ab-initio total-energy calculations using a plane-wave basis set. Phys. Rev. B54: 11169-11186.

Kresse G., Joubert D., 1999. From ultrasoft pseudopotentials to the projector augmentedwave method. Phys Rev B, 59, 1758-1775.

Laio, A., Bernard, S., Chiarotti, G.L., Scandolo, S., and Tosatti, E., 2000. Physics of iron at Earth's core conditions. Science. 287: 1027-1030.

Lehmann, I., 1936. P'. Trav. Sci. Sect. Seis. U.G.G.I. (Toulouse), 14, 3-31.

Lin, J-F., Heinz, D. L., Campbell, A. J., Devine, J. M., and Shen, G., 2002. Iron-silicon alloy in Earth's core? Science 295: 313-315.

Mao, H.K., Shu, J., Shen, G., Hemley, R.J., Li, B., and Sing, A.K., 1999. Elasticity and rheology of iron above 220GPa and the nature of the Earth's inner core. Nature. 399: 280.

Mao, H.K., Xu, J., Struzhkin, V.V., Shu, J., Hemley, R.J., Sturhahn, W., Hu, M.Y., Alp, E.E., Vočadlo, L., Alfè, D., Price, G.D., Gillan, M.J., Schwoerer-Bohning, M., Hausermann, D., Eng, P., Shen, G., Giefers, H., Lubbers, R., and Wortmann, G., 2001. Phonon density of states of iron up to 153 gigapascals. Science. 292: 914-916.

Masters, G., and Gubbins, D., 2003. On the resolution of density within the Earth. 
Phys. Earth Planet. In., 140: 159-167.

Matsui, M., and Anderson, O.L., 1997. The case for a body-centered cubic phase for iron at inner core conditions. Physics Earth Planetary Interiors. 103: 55-62.

McDonough, W.F., and Sun, S.-S., 1995. The composition of the Earth. Chem. Geol. 120: 223253.

Nguyen, J.H., and Holmes, N.C., 1999. Iron sound velocities in shock wave experiments. Shock Compression of Condensed Matter. CP505: 81-84.

Oldham, R.D., 1906. The constitution of the Earth as revealed by earthquakes. Quart. J. Geol. Soc., 62, 456-75

Oganov, A.R., Brodholt, J.P., Price, G.D., 2001. The elastic constants of $\mathrm{MgSiO}_{3}$ perovskite at pressures and temperatures of the Earth's mantle. Nature, 411, 934- 937

Parker, S.C., and Price, G.D., 1989. Computer Modelling of Phase Transitions in Minerals. Advances in Solid State Chemistry. 1: 295-327.

Parrinello, M., and Rahman, A., 1980. Crystal Structure and Pair Potentials: A Molecular Dynamics Study. Phys. Rev. Lett. 45: 1196-1199.

Poirier, J.P., 1994a. Light elements in the Earth's outer core: A critical review. Phys. Earth Planet. Inter. 85: 319-337.

Poirier, J.P., 1994b. Physical-properties of the Earths core. Cr. Acad. Sci. II. 318: 341-350.

Poirier, J.P., and Shankland, T.J., 1993. Dislocation Melting Of Iron And The Temperature Of The Inner-Core Boundary, Revisited. Geophys. J. Int., 115, 147-151.

Ross, M., Young, D. A., and Grover, R., 1990. Theory of the iron phase diagram at Earth core conditions. J. Geophys. Res. 95, 21,713-21,716.

Saxena, S.K., Dubrovinsky, L.S., and Häggkvist, P., 1996. X-ray evidence for the new phase of $\beta$-iron at high temperature and high pressure. Geophys. Res. Lett. 23: 2441-2444.

Shearer, P.M., and Masters, G., 1990. The density and shear velocity contrast at the inner core boundary. Geophys. J. Int., 102, 491-498.

Scandolo, S., 2002. First-principles molecular dynamics simulations at high pressure. Proc. of the International School of Physics "E. Fermi" on "High Pressure Phenomena", Course CXLVII (IOS, Amsterdam), 195-214.

Shen, G.Y., and Heinze, D.L., 1998. High Pressure melting of deep mantle and core materials. In: Ultrahigh-pressure Mineralogy (ed. R.J. Hemley). Reviews in Mineralogy. 37: 369-398.

Shen, G.Y., Mao, H.K., Hemley, R.J., Duffy, T.S., and Rivers, M.L., 1998. Melting and crystal structure of iron at high pressures and temperatures. Geophys. Res. Lett. 25: 373.

Söderlind, P., Moriarty, J.A., and Wills, J.M., 1996. First-principles theory of iron up to earth-core pressures: Structural, vibrational and elastic properties. Phys. Rev. B53:1406314072.

Song, X.D., 1997. Anisotropy of the Earth's Inner core. Reviews Geophys., 35, 297-313.

Song, X. D., and Helmberger, D. V., 1998. Seismic evidence for an inner core transition zone. Science, 282, 924-927.

Steinle-Neumann, G., Stixrude, L., and Cohen, R.E., 1999. First-principles elastic constants for the hcp transition metals Fe, Co, and Re at high pressure. Phys. Rev. B60: 791-799.

Steinle-Neumann, G., Stixrude, L., and Cohen, R.E., 2003. Physical properties of iron in the inner core. In: Earth's Core (eds. V. Dehant, K.C. Creager, S-i. Karato, S. Zatman). AGU, Geodynamics Series, 31, 137-162.

Steinle-Neumann, G., Stixrude, L., Cohen, R.E., and Gülseren, O., 2001. Elasticity of iron at the temperature of the Earth's inner core. Nature. 413: 57-60.

Stixrude, L., and Brown, J.M., 1998. The Earth's Core. In: Ultrahigh-pressure Mineralogy 
(ed. R.J. Hemley). Reviews in Mineralogy. 37: 261-283.

Stixrude, L., and Cohen, R.E., 1995. Constraints on the Crystalline Structure of the Inner Core - Mechanical Instability of $b c c$ Iron at High-Pressure. Geophys. Res. Lett. 22: 125-128.

Stixrude, L., Cohen, R.E., and Hemley, R.J., 1998. Theory of minerals at high pressure. In: Ultrahigh-pressure Mineralogy (ed. R.J. Hemley). Reviews in Mineralogy. 37: 639-671.

Stixrude, L., Wasserman, E., and Cohen, R.E., 1997. Composition and temperature of the Earth's inner core. J Geophys. Res. 102: 24729-24739.

Vočadlo, L., and Alfè, D., 2002. The ab initio melting curve of aluminum. Phys. Rev. B65: 214105, 1-12.

Vočadlo, L., Alfè, D., Gillan, M.J., Wood, I.G., Brodholt, P.J., and Price, G.D., 2003. Possible thermal and chemical stabilisation of body- centred- cubic iron in the Earth's core? Nature, 424, 536-53

Vočadlo, L., Brodholt, J., Alfè, D., Price, G.D., and Gillan, M.J., 1999. The structure of iron under the conditions of the Earth's inner core. Geophys. Res. Lett. 26: 1231-1234.

Vočadlo, L., deWijs, G.A., Kresse, G., Gillan, M.J., and Price, G.D., 1997. First principles calculations on crystalline and liquid iron at Earth's core conditions. Faraday Discussions. 106: 205-217.

Wasserman, E., Stixrude, L., and Cohen, R.E., 1996. Thermal properties of iron at high pressures and temperatures. Phys. Rev. B53: 8296-8309.

Wentzcovitch, R.M., 1991.Invariant Molecular-Dynamics Approach To Structural PhaseTransitions. Phys. Rev. B, 44: 2358-2361.

de Wijs, G.A., Kresse, G., and Gillan, M.J., 1998. First order phase transitions by first principles free energy calculations: The melting of Al. Phys. Rev. B57: 8233-8234.

Yoo, C.S., Holmes, N.C., Ross, M., Webb, D.J., and Pike, C., 1993. Shock temperatures and melting of iron at Earth core conditions. Phys. Rev. Lett. 70: 3931-3934. 
Table 1: The ab initio Helmholtz free energy per atom of the $b c c$ and $h c p$ phases of Fe at state points along (*and below) the calculated melting curve. In this work, the reference system is a simple inverse power potential which takes the form $U=4 \varepsilon(\Gamma / \mathrm{r})^{\alpha}$ where $\varepsilon=1 \mathrm{eV}$, $\Gamma=1.77 \AA, \alpha=5.86$. We have previously shown that this reference potential, based on only a repulsive term, describes the $a b$ initio system extremely well; the bonding term is almost independent of the atomic positions, depending only on the volume and temperature of the system. We stress, however, that the final result is totally independent of the choice of reference system. We performed these calculations on a 64 atom supercell $(4 \times 4 \times 4$ primitive cells) with a $3 \times 3 \times 3$ k-point grid. Considerable effort was spent on convergence tests in kpoint sampling to reduce the error in free energies to $<10 \mathrm{meV}$ per atom. Cell size effects have been extensively studied in our previous work hcp-Fe (Alfè et al., 2001).

\begin{tabular}{|l|l|l|l|l|}
\hline $\mathbf{V}\left(\AA^{\mathbf{3}}\right)$ & $\mathbf{T}(\mathbf{K})$ & $\mathbf{F}_{\text {bcc }}(\mathbf{e V})$ & $\mathbf{F}_{\text {hcp }}(\mathbf{e V})$ & $\begin{array}{l}\Delta \mathbf{F}_{\text {bcc-hcp }} \\
(\mathbf{m e V})\end{array}$ \\
\hline $\mathbf{9 . 0}$ & 3500 & -10.063 & -10.109 & 46 \\
\hline $\mathbf{8 . 5}$ & 3500 & -9.738 & -9.796 & 58 \\
\hline $\mathbf{7 . 8}$ & 5000 & -10.512 & -10.562 & 50 \\
\hline $\mathbf{7 . 2}$ & 6000 & -10.633 & -10.668 & 35 \\
\hline $\mathbf{6 . 9}$ & 6500 & -10.545 & -10.582 & 37 \\
\hline $\mathbf{6 . 7}$ & 6700 & -10.288 & -10.321 & 33 \\
\hline$* 7.2$ & 3000 & -7.757 & -7.932 & 175 \\
\hline
\end{tabular}




\section{Figure Captions}

Fig 1. A hypothetical phase diagram for Fe, incorporating all the experimentally suggested high $\mathrm{P} / \mathrm{T}$ phase transformations.

Fig 2. Schematic phase diagram for Fe based on the results of Vočadlo et al. (1999).

Fig 3. Our calculated high-pressure melting curve for $\mathrm{Al}$ (see Vočadlo and Alfè, 2002) is shown passing through a variety of recent high $\mathrm{P}$ experimental points.

Fig 4. Our calculated high $\mathrm{P}$ melting curve of Fe (plotted as a solid black line) is shown passing through the shock wave datum (open blue circle) of Brown and McQueen (1986). Other data shown includes: our melting curve corrected for the GGA pressure error (black dashed line), Belonoshko's melting curve (blue line), Belonoshko's melting data corrected for errors in potential fitting (black dots), Laio et al's melting curve (green line), Boehler's DAC curve (green dashed line), Shen's data (green diamonds), Yoo's shock data (open squares), William's melting curve (pale green dashed line).

Fig 5. Liquid and solid impurity mole fractions $\mathrm{c}_{\mathrm{x}}{ }^{1}$ and $\mathrm{c}_{\mathrm{x}}{ }^{\mathrm{s}}$ of impurities $\mathrm{X}=\mathrm{S}, \mathrm{Si}$ and $\mathrm{O}$, and resulting densities of the inner and outer core predicted by ab initio simulations. Solid, dashed and chain curves represent $\mathrm{S}, \mathrm{Si}$ and $\mathrm{O}$ respectively. (a) liquid density $\rho^{1}\left(\mathrm{kgm}^{-3}\right)$; horizontal dotted line shows density from seismic data. (b) mole fractions in solid resulting from equality of chemical potentials in solid and liquid. (c) relative density discontinuity $\left(\delta \rho / \rho^{1}\right)$ at the ICB; horizontal dotted line is the value from free oscillation data.

Fig 6. Plot of bulk modulus (diamonds) and shear modulus (squares) for hcp Fe as a function of pressure, with values taken from Stixrude and Cohen (1995), Steinle-Neumann et al (1999), Söderlind et al (1996), Mao et al. (1999) and Vočadlo et al. (2003). Black diamonds and squares represent $a b$ initio values, while white diamonds and squares represent values obtained from experimentally determined elastic constants.

Fig 7. Plot of aggregate $v_{P}$ (diamonds) and $v_{S}$ (squares) wave velocity for $h c p$ Fe as a function of pressure, with values taken from Stixrude and Cohen (1995), Steinle-Neumann et al (1999), Söderlind et al (1996), Mao et al. (1999) and Vočadlo et al. (2003). Black diamonds and squares represent $a b$ initio values, while white diamonds and squares represent values obtained from experimentally determined elastic constants. White circles are the experimental data of Fiquet et al. (2001). 
Figure 1

Temp

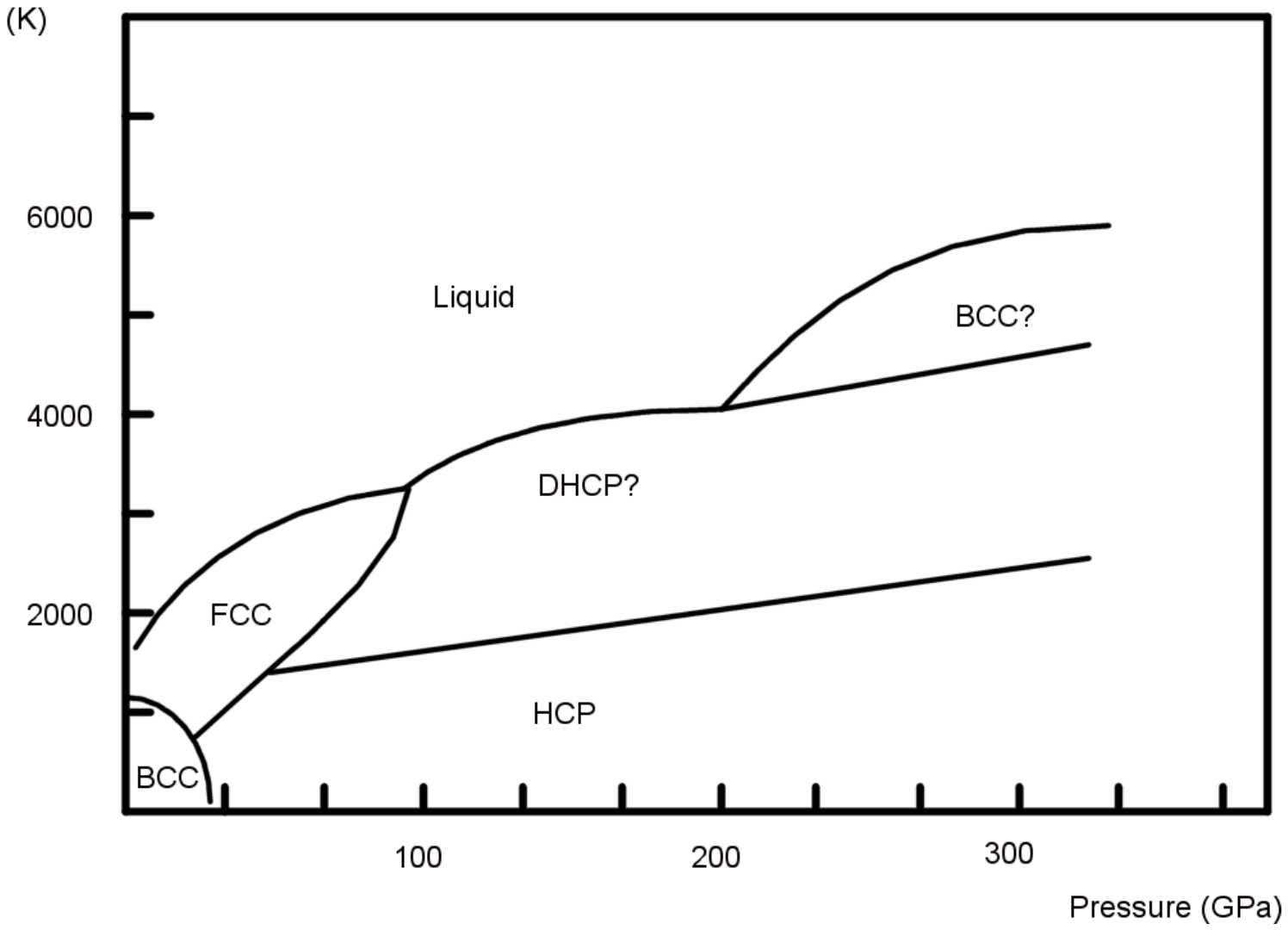


Figure 2

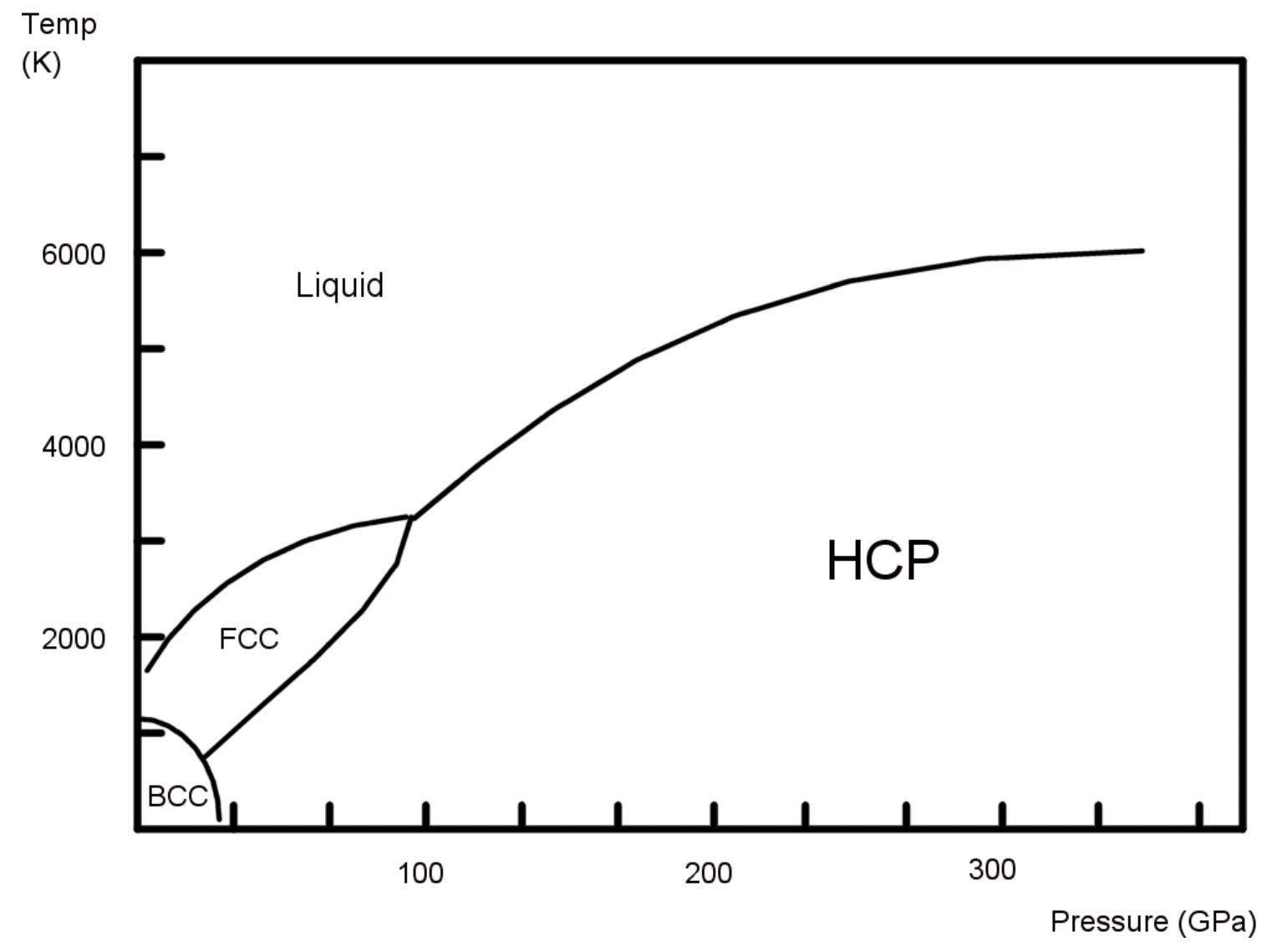


Figure 3

\section{$\boldsymbol{A} \boldsymbol{b}$ Initio Melting Curve of Aluminium}

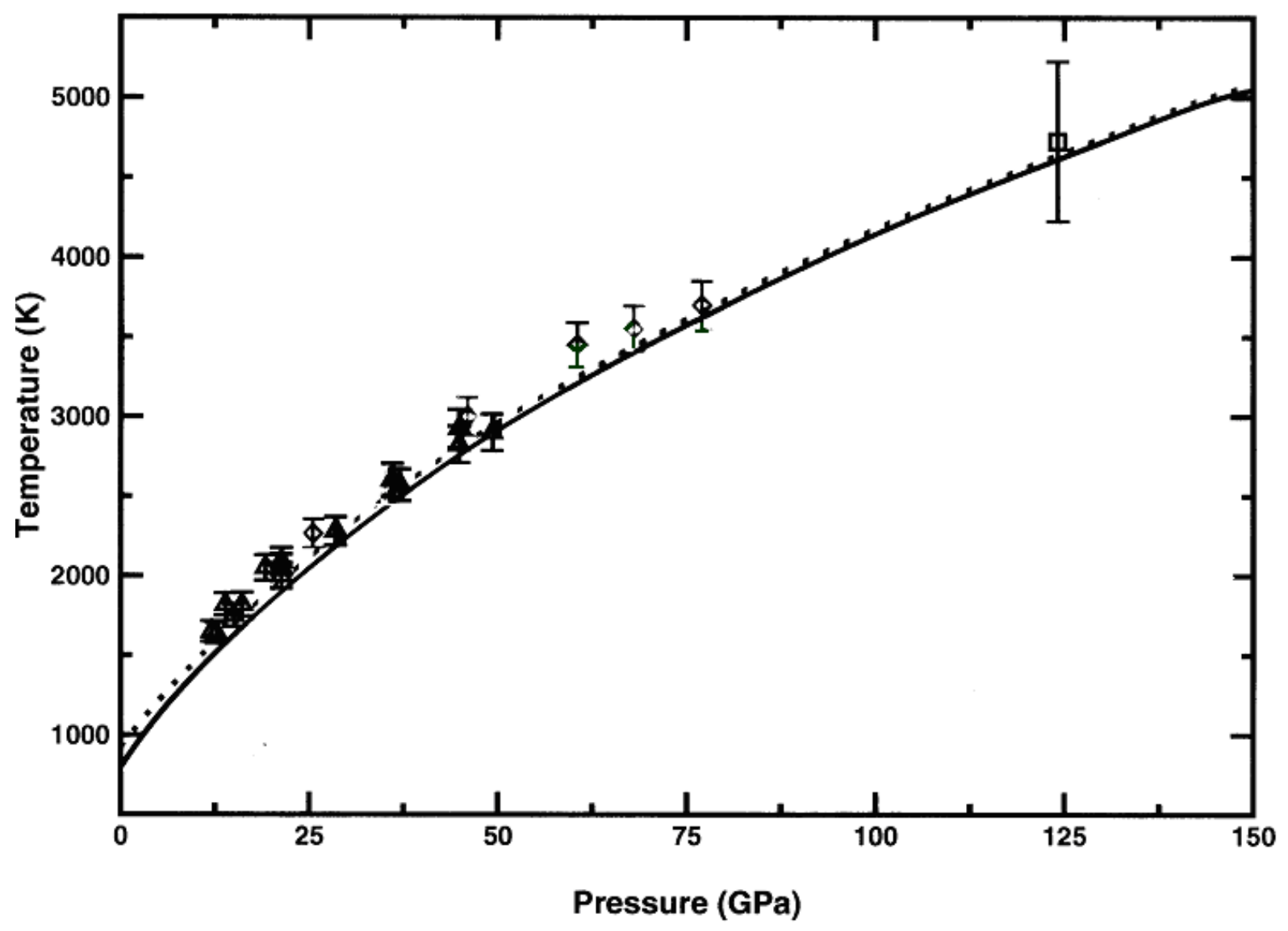


Figure 4

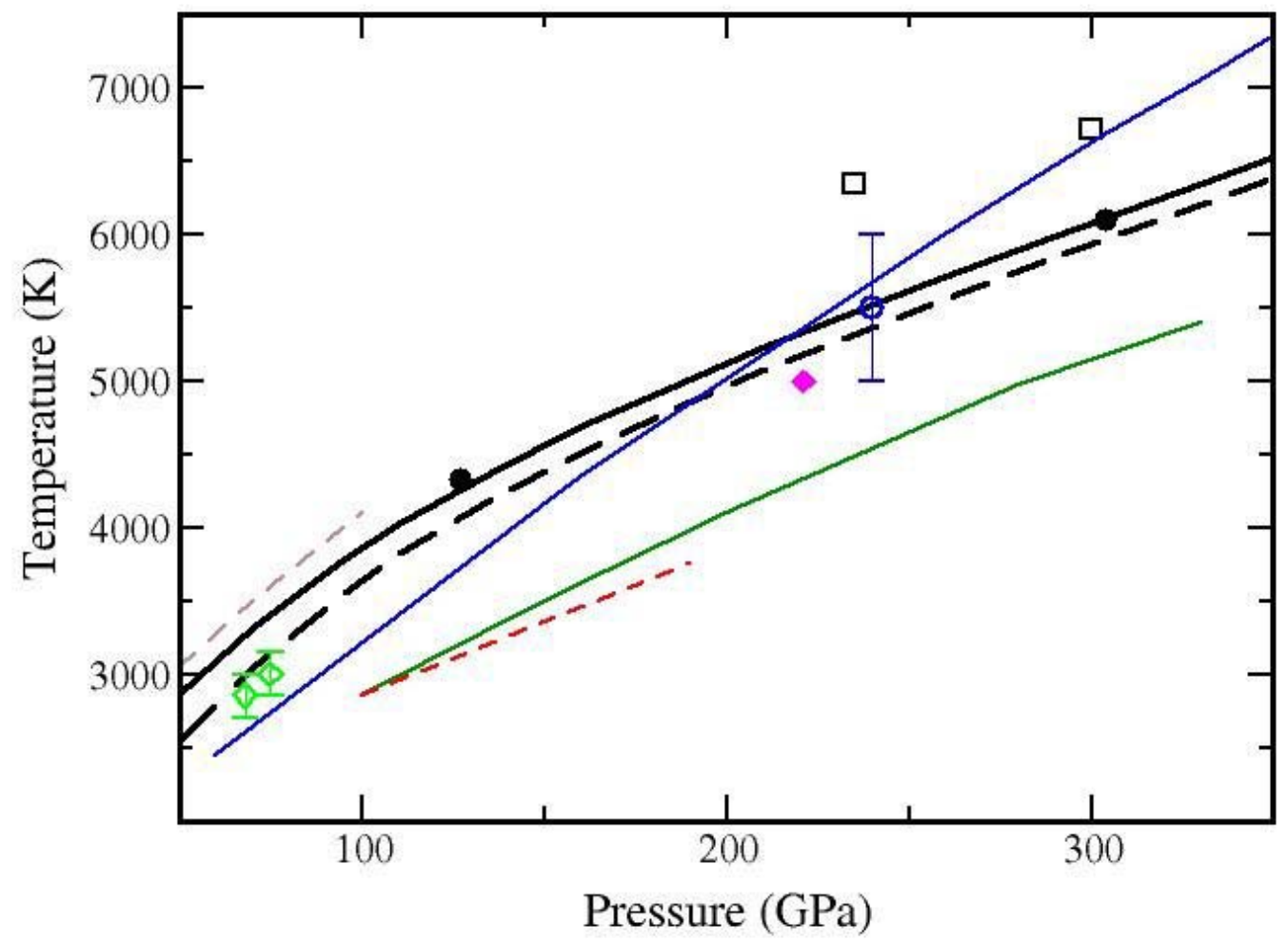


Figure 5
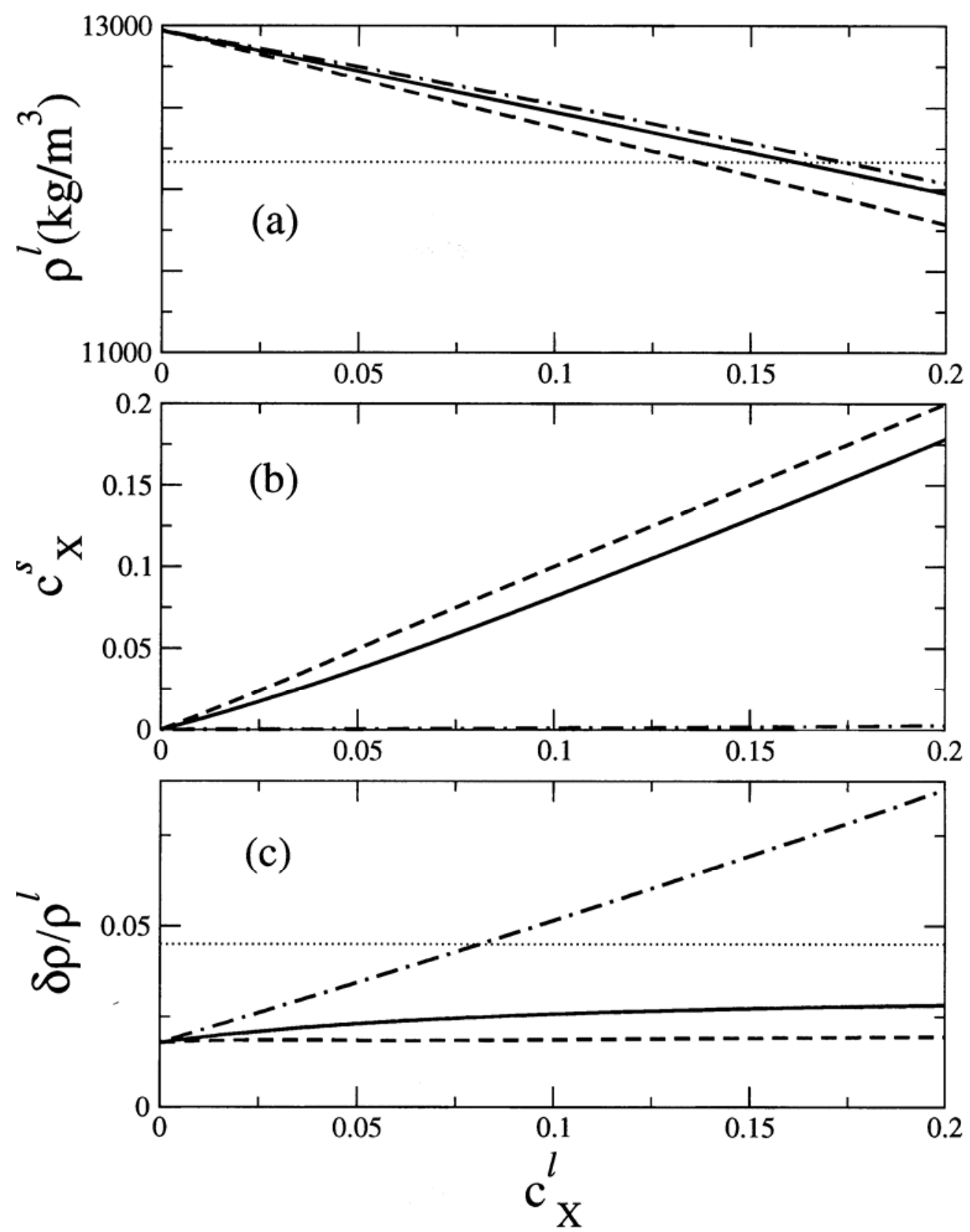
Figure 6

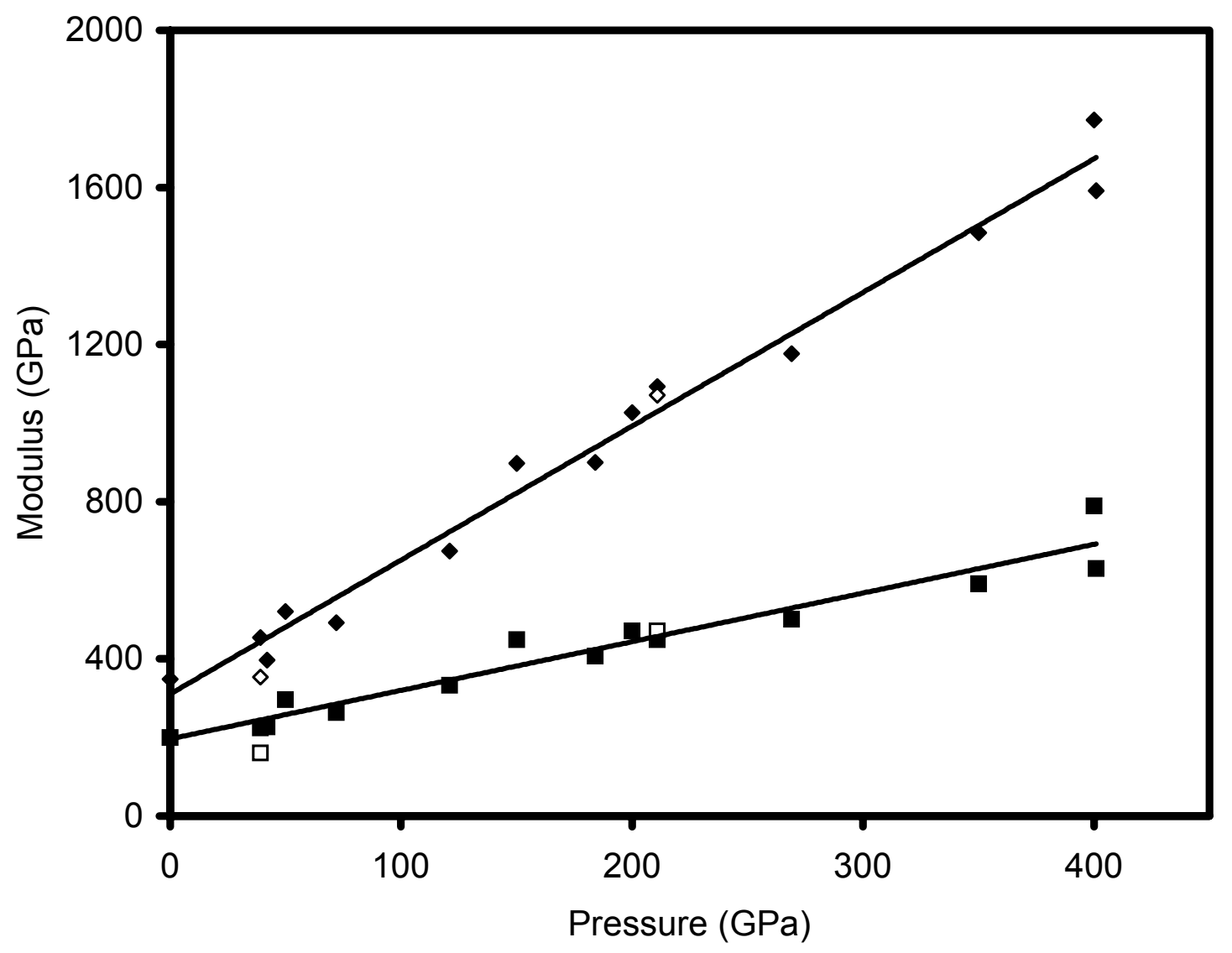


Figure 7

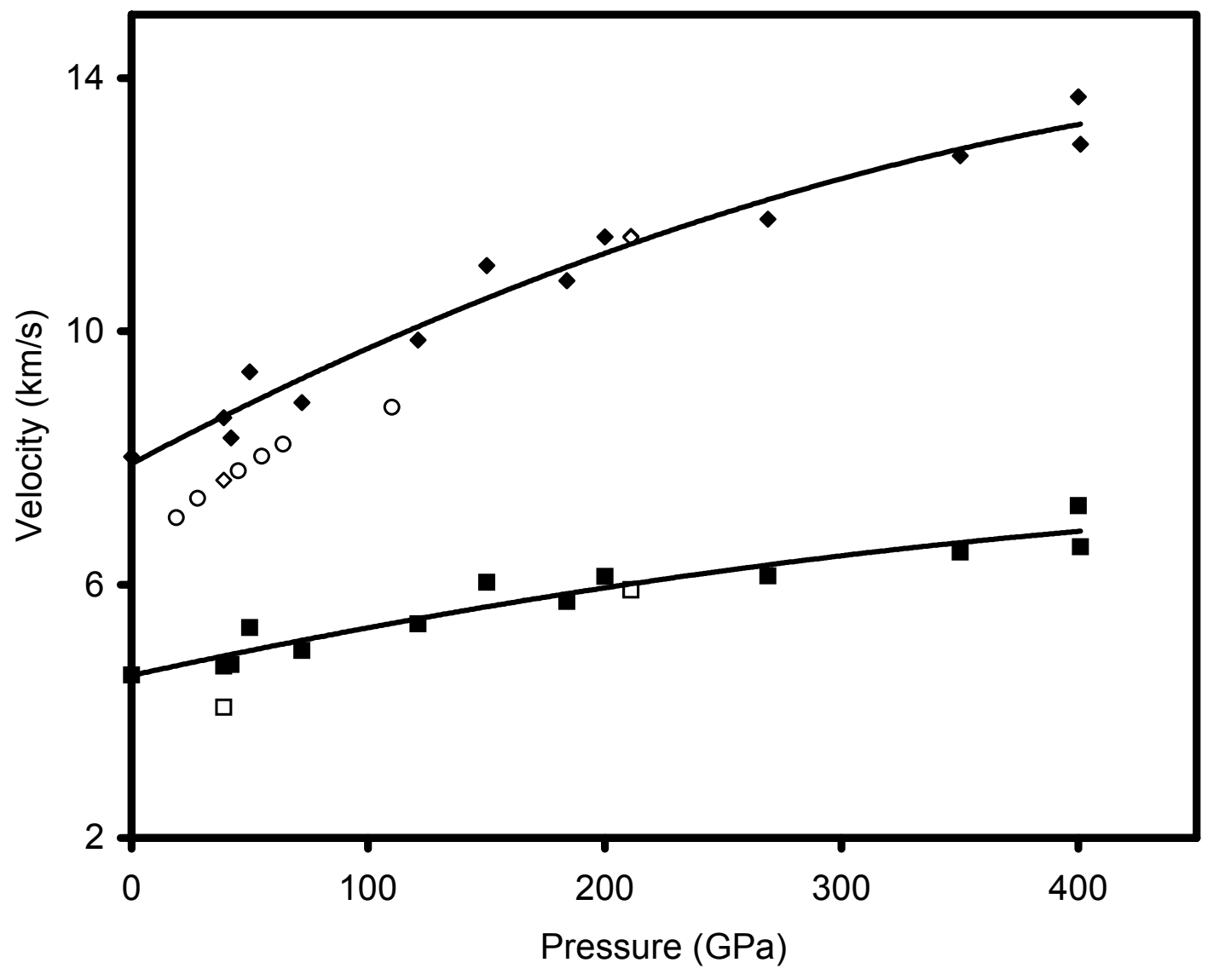

